\title{
THE
}

\section{High-Field Specific-Heat and Susceptibility Measurements: Relevance to the Spin-Peierls Phase Diagram and the Validity of a Soliton Picture}

\author{
J. C. Bonner \\ University of Rhode Island \\ J. A. Northby \\ University of Rhode Island, jnorthby@uri.edu \\ I. S. Jacobs \\ L. V. Interrante
}

Follow this and additional works at: https://digitalcommons.uri.edu/phys_facpubs

Terms of Use

All rights reserved under copyright.

\section{Citation/Publisher Attribution}

Bonner, J. C., Northby, J. A., Jacobs, I. S., \& Interrante, L. V. (1987). High-field specific-heat and susceptibility measurements: Relevance to the spin-Peierls phase diagram and the validity of a soliton picture. Physical Review B, 35(4), 179-1798. doi: 10.1103/PhysRevB.35.1791

Available at: http://dx.doi.org/10.1103/PhysRevB.35.1791

This Article is brought to you for free and open access by the Physics at DigitalCommons@URI. It has been accepted for inclusion in Physics Faculty Publications by an authorized administrator of DigitalCommons@URI. For more information, please contact digitalcommons-group@uri.edu. 


\title{
High-field specific-heat and susceptibility measurements: Relevance to the spin-Peierls phase diagram and the validity of a soliton picture
}

\author{
J. C. Bonner and J. A. Northby \\ Physics Department, University of Rhode Island, Kingston, Rhode Island 02881 \\ I. S. Jacobs and L. V. Interrante* \\ General Electric Company, Corporate Research and Development, Schenectady, New York 12301
}

(Received 23 June 1986)

\begin{abstract}
We discuss recent high-field specific-heat measurements on the spin-Peierls compound tetrathiafulvalinium bis-cis-(1,2-perfluoromethylethylene-1-2-dithiolato)-gold [TTF-BDT(Au)]. An ordering anomaly can clearly be detected which defines a high-field phase boundary, despite some extraneous structure in the data apparently reflecting experimental limitations. The high-field phase boundary is in good quantitative agreement with the theory of Cross and Fisher. Further, the specific-heat data are in good agreement with earlier ac susceptibility data, the reliability of which as indicators of a phase transition has been somewhat open to doubt because pronounced relaxation effects in the high-field regime result in striking differences between $\chi_{\mathrm{ac}}$ and $\chi_{\mathrm{dc}}$. Some observations are made concerning the extent of hysteresis associated with the phase boundary between dimerized and high-field phases. Finally, the extensive experimental data are employed in several tests to determine the applicability of recent soliton theories.
\end{abstract}

\section{INTRODUCTION}

The compound tetrathiafulvalinium bis-cis-(1,2perfluoromethylethylene-1-2-dithiolato)-gold [TTF$\mathrm{AuS}_{4} \mathrm{C}_{4}\left(\mathrm{CF}_{3}\right)_{4}$, denoted TTF-BDT(Au)] is one of the very few experimental examples of a spin-Peierls (SP) system. Such systems show a zero-field transition in which an assembly of spin- $\frac{1}{2}$ Heisenberg antiferromagnetic uniform chains experience a progressive dimerization as the temperature is lowered through the spin-Peierls transition temperature, $T_{\mathrm{SP}}(0)$. This transition is a consequence of magnetoelastic coupling of the spin chains to the threedimensional (3D) lattice. The three compounds that have received extensive experimental investigation are TTFBDT $(\mathrm{Au})$, where $T_{\mathrm{SP}}(0) \sim 2 \mathrm{~K}$; its sister compound TTF$\operatorname{BDT}(\mathrm{Cu})$, where $T_{\mathrm{SP}}(0) \sim 11 \mathrm{~K} ;$ and the compound methylethylmorpholinium ditetracyanoquinodimethane [MEM-(TCNQ) $)_{2}$ ], where $T_{\mathrm{SP}}(0) \sim 18 \mathrm{~K} .{ }^{1}$

The preparation of good samples is very difficult in the case of the TTF-BDT compounds, and for all three compounds experimental difficulties preclude a precise location of $T_{\mathrm{SP}}(0)$. Hence, at present, the nature of the critical exponents characterizing the novel spin-Peierls transition cannot be determined. However, the global phase diagram is of great interest. The ordering behavior of the SP compounds is the outcome of competition between a spin-phonon interaction (which gives rise to an SP transition) and spin-spin interchain coupling (which would result in regular 3D antiferromagnetic ordering). An applied magnetic field will influence the relative importance of these two competing phase mechanisms, ${ }^{2-4}$ and hence novel field-induced phase transitions are expected.

The majority of extant theoretical approaches qualitatively predict an $(H, T)$ phase diagram as shown in Fig.
1. ${ }^{1}$ The major features of this sketch include a dimerized phase region denoted $\mathrm{D}$, extending in field up to a special value $H^{*}$, a paramagnetic region, denoted $\mathrm{U}$, where the system behaves as an assembly of noninteracting uniform Heisenberg antiferromagnetic chains, and a high-field phase denoted I. The DU and IU phase boundaries are predicted to be higher order (second order) but there are conflicting predictions for the DI phase boundary. Those theories predicting first-order character suggest also that the DI transition line will be characterized by a notable amount of hysteresis extending over an appreciable range

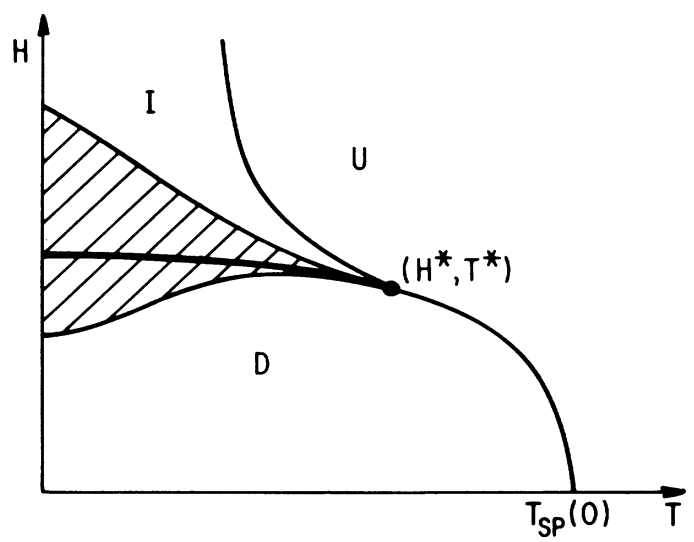

FIG. 1. Schematic $(H-T)$ phase diagram of a spin-Peierls system indicating dimerized (D), uniform (U), and intermediate (incommensurate) (I) phases. The DU and IU phase boundaries are second order and $\left(H^{*}, T^{*}\right)$ is a Lifshitz point. The DI line, believed to be first order, is shown with an associated region of hysteresis (shaded) (after de Lima and Tsallis, Ref. 23).

(C) 1987 The American Physical Society 
in the $(H, T)$ plane. The DU, DI, and IU phase boundaries meet at a special multicritical point $\left(H^{*}, T^{*}\right)$, which is expected to be a Lifshitz point.

Existing magnetization, ${ }^{2,5-7}$ susceptibility, ${ }^{8}$ and specific-heat ${ }^{9}$ data for TTF-BDT(Au), TTF-BDT $(\mathrm{Cu})$, and MEM-(TCNQ $)_{2}$ are in agreement with Fig. 1, at least as far as the DU phase boundary is concerned. Experiment is in support of the first-order character predicted for the DI phase boundary. Further, if the $H$ and $T$ axes are both scaled by $T_{\mathrm{SP}}(0)$, i.e., if the data are plotted as a function of $H / T_{\mathrm{SP}}(0)$ and $T / T_{\mathrm{SP}}(0)$, measurements on all three compounds closely superimpose. This indicates that the $(H, T)$ spin-Peierls phase diagram has universal character, ${ }^{8}$ as expected on the basis of standard spinPeierls theory. ${ }^{1}$ Furthermore, extensive hysteresis in the neighborhood of the DI line, particularly at low temperature, observed in TTF-BDT $(\mathrm{Cu})$ (Refs. 4-6) and MEM$(\mathrm{TCNQ})_{2}$ (Ref. 7) has very recently been observed also in TTF-BDT $(\mathrm{Au}){ }^{10}$

Important universal parameters for points in the phase diagram are found experimentally to be $H^{*} / T_{\mathrm{SP}}(0) \sim 1.0$ ( $H$ in tesla and $T$ in $\mathrm{K}$ ), and $T^{*} / T_{\mathrm{SP}}(0) \sim 0.75$. Data delineating the IU line in Ref. 8 were obtained from magnetization measurements (on all three compounds) and ac susceptibility measurements [TTF-BDT $(\mathrm{Au})$ only]. We note that of the three compounds, measurements over a substantial field range above $H^{*}$ are feasible only for TTF$\operatorname{BDT}(\mathrm{Au})$. Since TTF-BDT $(\mathrm{Au})$ has the lowest value for $T_{\text {SP }}(0)$ (taken to be $2.03 \mathrm{~K}$ ), by the universal scaling properties, the corresponding value of $H^{*}$ is expected to be about $2 \mathrm{~T}$. Hence, the existence and nature of a high-field phase can be probed most easily in the case of TTF$\operatorname{BDT}(\mathrm{Au})$, and with greater difficulty in the case of TTF$\operatorname{BDT}(\mathrm{Cu})\left(H^{*} \sim 11.5 \mathrm{~T}\right)$ and $\mathrm{MEM}-(\mathrm{TCNQ})_{2}\left(H^{*} \sim 18\right.$ T).

Experimental results on TTF-BDT(Au) for differential (ac) susceptibility have already been reported, which allowed determination of DU and DI phase boundaries and an appreciable portion of the IU phase boundary. ${ }^{8}$ Subsequent specific-heat measurements at low fields ${ }^{9}$ were in excellent agreement with the susceptibility data in defining the DU boundary. A notable feature of the susceptibility measurements was the pronounced relaxation effects in the high-field region. When the ac susceptibility results were compared with corresponding static, isothermal susceptibility results obtained by differentiating magnetization isotherms, the two quantities $\chi_{\mathrm{ac}}$ and $\chi_{\mathrm{dc}}=\chi_{T}$, were equivalent along the DU boundary, indicating that relaxation effects were unimportant in the case of the dimerized-to-uniform transition. However, in the highfield phase region, including the DI and IU lines, $\chi_{\mathrm{ac}}$ and $\chi_{T}$ differ considerably, indicating that relaxation effects are prominent in this region of the phase diagram. This situation is shown in the inset of Fig 2. Figure 2 features ac susceptibility curves as a function of temperature for various field values both below and above $H^{*}(2.1 \mathrm{~T})$. The $\chi_{\mathrm{ac}}$ data exhibit a peak except at the lowest field values. In the high-field phase region (data points denoted by open symbols), for $H>2.1 \mathrm{~T}$, the peak in $\chi_{\mathrm{ac}}$ is not paralleled by a peak in $\chi_{\mathrm{dc}}=\chi_{T}$, the latter quantity continuing to increase as $T$ decreases. In consequence, the

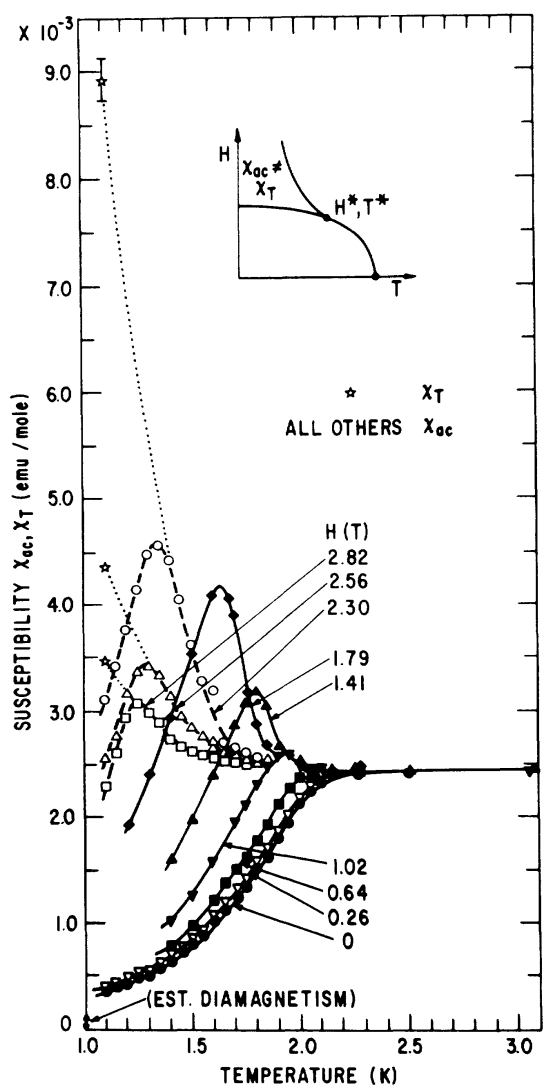

FIG. 2. Differential (ac) susceptibility curves, along with some isothermal susceptibility data, at various constant fields as a function of temperature for TTF-BDT(Au). Closed data points are for $H<H^{*}$ and open ones are for higher fields. The inset shows the $(H, T)$ region in which ac and dc (isothermal) susceptibilities are unequal.

question was raised whether the IU line determined from $\chi_{\text {ac }}$ measurements corresponded to a "true" phase boundary, or was just an artifact of relaxation effects. ${ }^{11,12}$ The relaxation effects were conjectured to result from a broad region in field of soliton nucleation processes associated with the (first-order) DI line. ${ }^{11,12}$ Since the specific heat is intrinsically a static quantity, and, therefore, independent of relaxation effects, anomalies in the specific heat are arguably the most reliable indicators of the existence of a phase transition. Hence, low-field specific-heat measurements ${ }^{9}$ were extended up to $4.0 \mathrm{~T}$; i.e., well above the critical field $H^{*} \sim 2.1 \mathrm{~T}^{13}$

\section{EXPERIMENTAL SITUATION}

Specific-heat data obtained in zero field and in fields of 2.0, 2.3, 2.6, and 4.0 T are shown in Fig. $3 .{ }^{13}$ The data for $H<2.2 \mathrm{~T}$ relate to the low-field DU phase boundary and the data for $H>2.2 \mathrm{~T}$ relate to the high-field IU phase boundary. In all cases a field-dependent, cusplike anomaly is observed, indicated by the arrows. The amplitude of the anomaly shows a steady decrease with increasing field as would be expected on theoretical grounds. The specific-heat peak for $H<H^{*}$ is in excellent agreement with earlier specific-heat studies. ${ }^{9}$ In the data for $H>0$, 


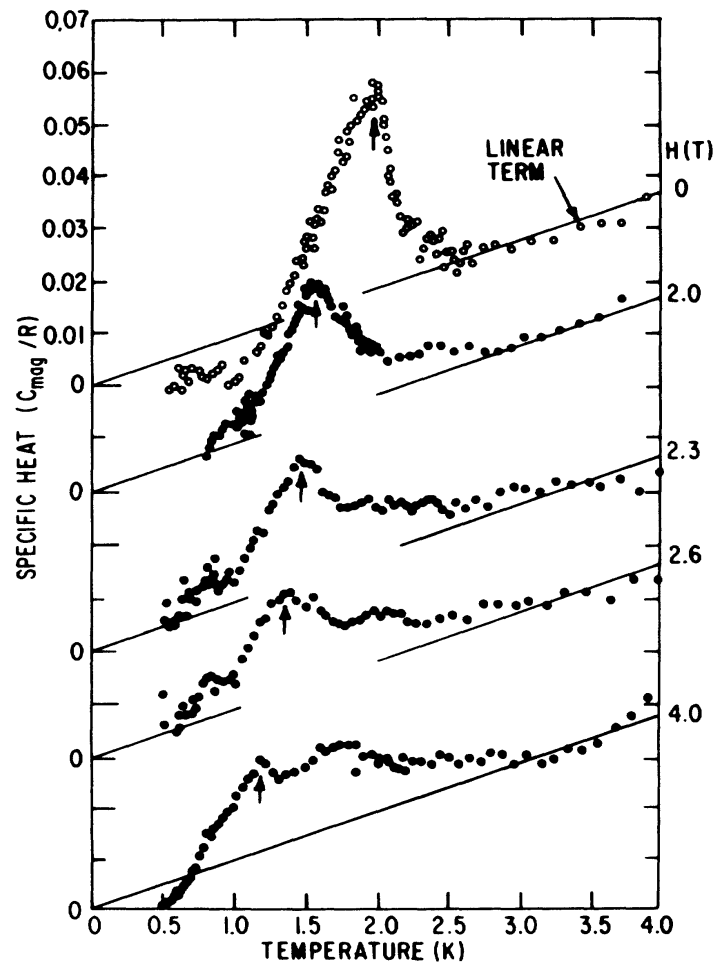

FIG. 3. Magnetic contributions to the specific heat in zero field and in various high fields $H \geq H^{*}$ for TTF-BDT(Au). The arrows indicate the field-dependent cusplike anomaly (after Hijmans et al., Ref. 13).

anomalous structure is observed at about $2.4 \mathrm{~K}$, independent of $H$. In the case of the low-field data, this anomalous structure does not affect the location of the specific-heat peak, and, hence, we conclude it may safely be ignored for all $H$. In the data for $H>H^{*}$, additional anomalous structure becomes evident at about $1.7 \mathrm{~K}$, whose magnitude becomes comparable with the amplitude of the "true" specific-heat anomaly at the highest fields. In fact, this anomalous structure can also be discerned at the lower field of $2.0 \mathrm{~T}$ as a "shoulder" on the hightemperature side of the DU specific-heat peak. Both amplitude and location of this anomaly are approximately field independent and, hence, we again conclude this structure is a spurious artifact. Overall, therefore, we conclude that anomalous structure appearing above the "true" specific-heat peak in temperature is most likely of extraneous contributions resulting from experimental procedures and not a property of the spin-Peierls system TTF-BDT $(\mathrm{Au})$. In particular, the fact that this extra structure is field independent implies that it does not result from, say, the effects of short-range order.

In Fig. 4 we show an $(H, T)$ phase diagram obtained on the basis of previous susceptibility and low-field specificheat measurements ${ }^{8,9}$ together with the new high-field specific-heat data. ${ }^{13}$ The DU line and part of the DI line are clearly delineated although a small amount of data scatter, reflecting the difficult experimental situation, is present. Three experimental data points define the IU phase boundary out to $H \sim 2 H^{*}$, and are in general agree-

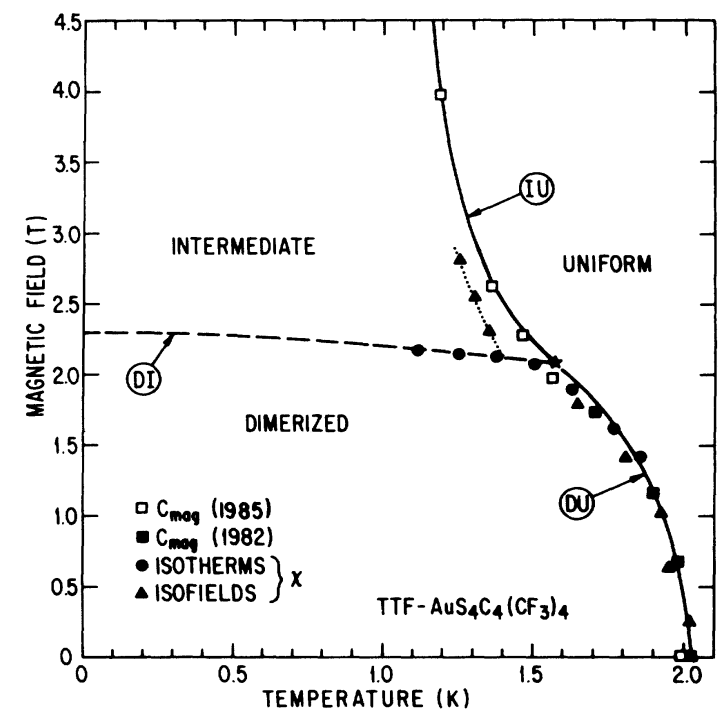

FIG. 4. Magnetic phase diagram for TTF-BDT(Au) conducted from susceptibility and specific-heat measurements (Refs. 8, 9, and 13) and the calculated phase boundaries (Ref. 16) scaled only by $T_{\mathrm{SP}}(0)$.

ment with previous IU data points obtained from ac susceptibility experiments. The agreement of two different types of experimental measurements indicates to us (a) that previous concerns over the reliability of determining a phase boundary from $\chi_{\mathrm{ac}}$ peaks in a situation where $\chi_{\mathrm{ac}} \neq \chi_{T}$ appear to be unfounded, and (b) that despite some spurious features apparently resulting from experimental artifacts, the new specific-heat data are reliably displaying an anomaly characterizing a phase transition from a paramagnetic (uniform) phase to a high-field phase (denoted "intermediate" on Fig. 4) of unspecified character.

It should be noted that our confidence in this interpretation of specific-heat and susceptibility data is strongly supported by very recent work consisting of frequencydependent proton and fluorine nuclear magnetic resonance (NMR) experiments on TTF-BDT(Au). ${ }^{10,13}$ Proton spinecho linewidth and relaxation studies define the IU phase boundary up to fields of about $3.3 \mathrm{~T}$, and the results are in excellent agreement with the specific-heat and ac susceptibility studies displayed in Fig. 4. The linewidth studies also extend the definition of the DI phase boundary from $1.1 \mathrm{~K}$ down to $0.57 \mathrm{~K}$ and reveal the presence of hysteresis below $\sim 1 \mathrm{~K}$, which confirms the first-order nature of the DI boundary.

\section{DISCUSSION}

\section{A. High-field phase boundary}

Let us examine the new experimental results in relation to theoretical calculations on spin-Peierls systems in a field. As noted in the Introduction, most theories predict a field-induced transition from a dimerized phase to an incommensurate ${ }^{14-20}$ or modulated (higher-order commensurate) phase. ${ }^{21-23}$ Such theories generally subdivide into 
soliton- and non-soliton-based theories. Other extant calculations predict that the high-field phase may correspond to regular three-dimensional antiferromagnetic ordering of either a spin-flop or antiferromagnetic (ordered Neel ground state) type, ${ }^{3,4}$ or even that no high-field phase is present. ${ }^{11,12}$ The experimental measurements reviewed here $^{13}$ and obtained from other work ${ }^{8,9}$ are in very good agreement with the theory of Cross and Fisher, ${ }^{16}$ amplified by Cross. ${ }^{16}$ The DU and IU phase boundaries of Cross are shown in Fig. 4, and very good quantitative agreement with the experimental data is observed. In particular, it is noteworthy that the agreement extends to the shape and location of the IU phase line (which is not the case for other theories of the IU phase boundary, ${ }^{15}$ ) and the location of the special multicritical point $\left(H^{*}, T^{*}\right)$ predicted by Cross to occur at $T^{*} / T_{\mathrm{SP}}(0) \sim 0.77$ and $\mu_{B} H^{*} / k_{B} T_{\mathrm{SP}}(0)=0.69(g=2.00)$. Both leading theories, that of Cross and Fisher, ${ }^{16}$ and the related soliton-lattice theory of Nakano and Fukuyama, ${ }^{20}$ predict the high-field phase to be incommensurate (but differ appreciably in their predictions for characteristic parameters related to the $(H, T)$ phase diagram as discussed below). Bulk thermodynamic (susceptibility and specific-heat) studies are not suited to determined whether the high-field phase is indeed an incommensurate phase. We can only note that the relatively weak IU specific-heat anomalies are consistent with current theoretical predictions ${ }^{24}$ concerning incommensurate or Kosterlitz-Thouless phases. The recent NMR work ${ }^{10}$ leads to calculations which demonstrate that the high-field phase is neither paramagnetic nor three-dimensionally ordered, either antiferromagnetically or in a spin-flop configuration. By these and other arguments related to agreement of certain features of the experiments with the Nakano-Fukuyama soliton-lattice picture, it is concluded that the high-field phase is, in fact, incommensurate. In the NMR work ${ }^{10}$ no attempt is made to compare the experimental data to a global phase diagram based on the Nakano-Fukuyama theory. One problem would be the fact that the field of the DI phase boundary, essentially equivalent to $H^{*}$, is given by $\mu_{B} H^{*} / k_{B} T_{\mathrm{SP}} \cong 0.28$, a value considerably lower than that resulting from the Cross theory and experiment.

\section{B. Susceptibility in the high-field phase}

A point of special interest is the striking difference between $\chi_{\mathrm{ac}}$ and the corresponding $\chi_{\mathrm{dc}}=\chi_{T}$ in the high-field phase region. This feature is shown in Fig. 5, where $\chi_{\mathrm{ac}}$ and $\chi_{T}$ are compared as a function of field at a temperature of $\sim 1.1 \mathrm{~K}^{8}{ }^{8}$ The maximum in both susceptibility curves occurs at the same field, $H \sim 2.2 \mathrm{~T}$, but the magnitude is considerably greater in the case of $\chi_{T}=d M_{T} / d H$. It should also be noted that there is noticeable asymmetry in the curves, particularly in the case of $\chi_{T}$. The curve rises sharply on the low-field side of the transition and drops more gradually on the high-field side. In an attempt to throw light on the high-field behavior of spinPeierls systems approximate calculations on a pure dimerized Heisenberg antiferromagnetic chain (without coupling to the lattice) were performed using the Bulaevski Hartree-Fock and related approaches. ${ }^{25}$ The qualitative

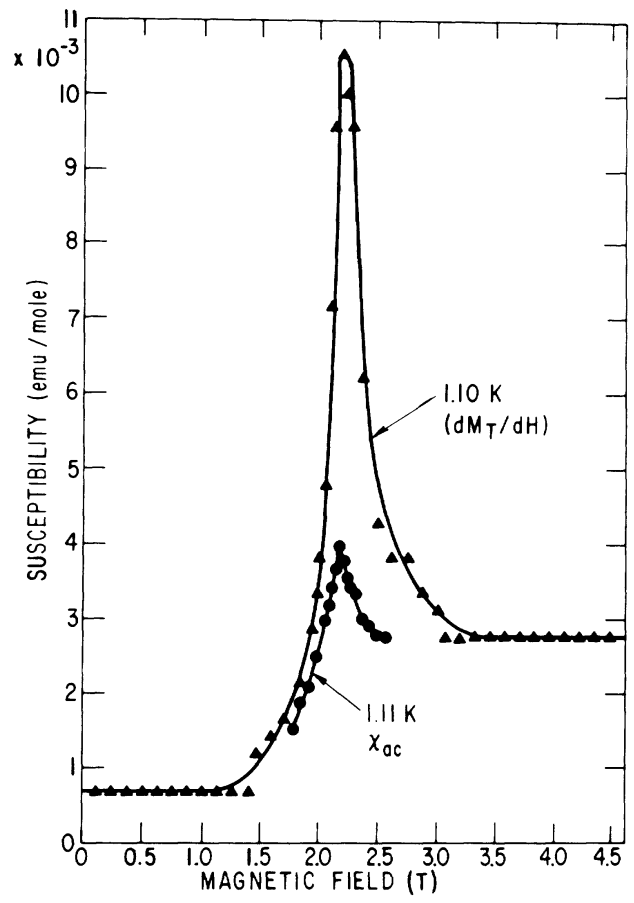

FIG. 5. Comparison of the field dependence of differential susceptibility, $\chi_{\mathrm{ac}}$, with the isothermal susceptibility, $\chi_{T}$, at $T=1.1 \mathrm{~K}$ for TTF-BDT(Au) (after Ref. 8).

accuracy of the results was checked by finite-chain calculations. ${ }^{26,27}$ In an alternating antiferromagnetic quantum system, the lowest excited states are triplets, and cross the zero-field singlet ground state as a result of Zeeman term effects at a lower critical field $H_{c 1}$. Results for the susceptibility, $\chi_{T}$, as a function of field for various low temperatures are shown as Fig. 2 of Ref. 25. The theoretical results in the vicinity of $H_{c 1}$ only, are shown schematically in Fig. 6. A pronounced peak occurs in the vicinity of $H_{c 1}$, which sharpens as $T$ is lowered, becoming divergent at $T=0$. (In the presence of interchain coupling, this divergence will be suppressed, but a pronounced "peaking" effect will remain.) Furthermore, an asymmetry of the kind observed experimentally is seen at low temperatures in the vicinity of $H_{c 1}$. A large dc susceptibility diverging as $T \rightarrow 0$ is characteristic of systems in which a region characterized by an energy gap changes to a gapless region due to the effect of a field. The continuous low-lying density of states gives rise to a power-law divergence of the susceptibility at $H=H_{c 1} \cdot{ }^{28}$ It also gives rise to a finite, nonzero value for the susceptibility for $H_{c 1}<H<H_{c 2}$, where $H_{c 2}$ is a second critical field at which a gap opens up. Hence it seems reasonable to conclude that similar phenomena will occur also for magnetoelastic systems where the spin-phonon coupling is weak $\left(T_{\mathrm{SP}}<J\right)$ and a dimerized phase with gaps shows a phase transition to a gapless (incommensurate) phase. Moving now from the vicinity of the DI phase boundary (Fig. 4), we discuss the expected behavior of $\chi_{\mathrm{ac}}$ and $\chi_{T}$ at constant field as a function of temperature in the vicinity of the IU phase boundary (see Fig. 2, inset). The ac suscepti- 


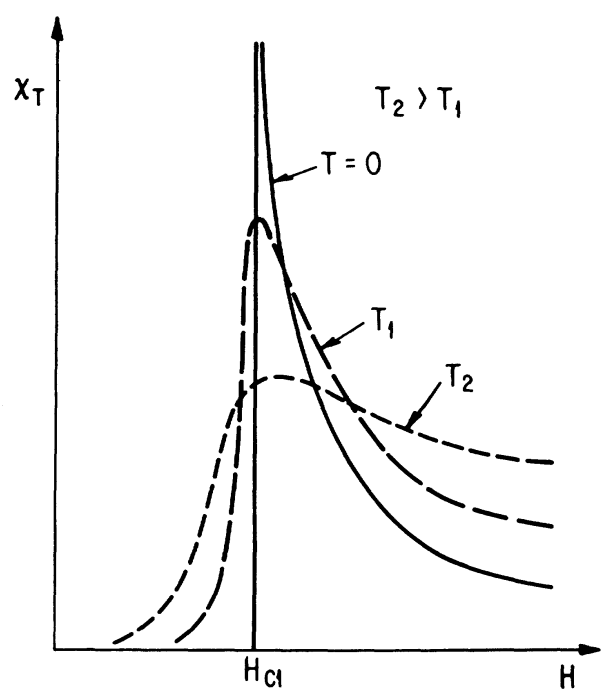

FIG. 6. Schematic plot of isothermal susceptibility, $\chi_{T}$, as a function of field at various low temperatures in the vicinity of a critical field, $H_{c 1}$. Note that the divergence at $T=0$ becomes a rounded peak at higher temperatures (after Mohan and Bonner, Ref. 25).

bility shows the peak characterizing the IU phase transition, while $\chi_{T}$ may show only a minimal anomaly, ${ }^{29}$ rising to large values in the neighborhood of $\left(H^{*}, T^{*}\right)$.

Recently a magnetoelastic calculation has indeed appeared, ${ }^{30}$ where, starting from the mean-field magnetoelastic Hamiltonian of Pytte, ${ }^{14}$ thermodynamic quantities such as magnetization, susceptibility, and specific heat have been calculated, using the soliton-lattice solution for the incommensurate state which takes into account infinite numbers of higher harmonics. ${ }^{19-21}$ This calculation supports the comment above that the inclusion of magnetoelastic coupling will not significantly change conclusions drawn from the purely magnetic calculation of Ref. 25. A plot of calculated dc susceptibility versus field at low temperatures, for example, shows a sharp rise at the low-field side of the DI phase boundary, and a more gradual drop to a nonzero value in the incommensurate phase above the DI boundary, in qualitative agreement with Fig. 6. More generally, the magnetization, susceptibility, and specific heat are qualitatively in agreement with experiment. ${ }^{8}$

\section{Hysteresis and the DI line}

The question of hysteresis along the DI lines is also of interest. In all cases, TTF-BDT $(\mathrm{Cu}), \mathrm{MEM}-(\mathrm{TCNQ})_{2}$, and TTF-BDT $(\mathrm{Au})$, the onset of hysteresis is observed not at the Lifshitz point $\left(H^{*}, T^{*}\right)$ but at significantly lower temperatures. In the case of TTF-BDT $(\mathrm{Cu})$ hysteresis is first observed in dc magnetization measurements at $T / T_{\mathrm{SP}}(0) \cong 0.50$; in $\mathrm{MEM}$ - $(\mathrm{TCNQ})_{2}$, also for dc magnetization measurements, at $T / T_{\mathrm{SP}}(0) \cong 0.6$; and in the case of TTF-BDT $(\mathrm{Au})$, for $T / T_{\mathrm{SP}}(0) \leq 0.5$ in spin-echo halfwidth measurements. ${ }^{10}$ Existing theories ${ }^{16,19,23}$ predict that the DI line is first order all the way up in temperature to the Lifshitz point. A situation where the DI line changes from first order to second order as the Lifshitz point is approached would be surprising. A possible resolution of this rather anomalous feature lies in a calculation by de Lima and Tsallis on magnetostrictive $X Y$ spin chains. $^{23}$ They show that in their model the DI line is associated with considerable hysteresis, as indicated in Fig. 1. The hysteretic region starts out near the Lifshitz point so narrow as to be effectively experimentally unobservable, and then "flares out" midway down the line. Such a theoretical prediction is certainly in accordance with current experimental observations.

Also of interest concerning the DI line is the apparent slope (see Fig. 4), which is negative. This forms an interesting contrast to the first-order antiferromagnetic-spin-flop phase boundary of antiferromagnets with some uniaxial anisotropy whose $(H, T)$ phase diagram is in some ways analogous to the $(H, T)$ phase diagram obtained for spin-Peierls systems.

\section{Validity of a soliton picture}

Our discussions above have focused on physical concepts and theories of the global spin-Peierls phase diagram which largely ignore nonlinear excitations and fluctuation phenomena. A case in point is our discussion of the behavior of $\chi_{\mathrm{ac}}\left(\chi_{T}\right)$ in the vicinity of the critical field. However, calculations based on a soliton picture are growing in popularity in comparison with theories based on the more traditional spin-exciton (magnon) picture. The magnetic model at low temperatures in the dimerized phase is that of the familiar antiferromagnetic alternating chain with alternation parameter $\delta$, where

$$
\mathscr{H}=\sum_{i=1}^{N}\left[J(1+\delta) \mathbf{S}_{2 i} \cdot \mathbf{S}_{2 i+1}+J(1-\delta) \mathbf{S}_{2 i-1} \cdot \mathbf{S}_{2 i}\right] .
$$

In the picture we have used, $\delta$ depends on temperature, $\delta=\delta(T)$, but at a given temperature, $\delta$ remains effectively constant along the chain. Soliton theories such as that of Nakano and Fukuyama ${ }^{20}$ essentially allow for fluctuations in $\delta$ (kink solitons). The Nakano-Fukuyama theory starts from the Cross-Fisher theory, where the spin-phonon coupling is treated in the random-phase approximation (RPA) and the magnetic chains are the basis of a Luttinger bosonization approach. ${ }^{16}$ The Hamiltonian is subsequently transcribed to phase variables and a sineGordon equation is obtained as a result of further approximations. The sine-Gordon equation is then solved for the lowest-lying excitations, which turn out to be solitons with effective spin $\frac{1}{2}$ instead of spin excitons (magnons) with effective spin 1. All existing formulations of soliton theory consistently predict that the soliton excitation energy, $\Delta E^{\text {sol }}$, is considerably lower than the magnon (spin exciton) creation energy, $\Delta E^{\mathrm{mag}}$. The approximate relation

$$
\Delta E^{\mathrm{sol}} \sim 0.3 \Delta E^{\mathrm{mag}}
$$

is obtained. ${ }^{31}$

Our analysis of the extensive experimental data available not only for TTF-BDT(Au), but also for TTF$\operatorname{BDT}(\mathrm{Cu})$ and $\mathrm{MEM}-(\mathrm{TCNQ})_{2}$, favors the more traditional spin-exciton over the popular soliton excitation picture. Specifically, we have performed three tests to distinguish 
between these two approaches:

(1) A comparison of theoretical predictions with experimental determinations of the characteristic features of the spin-Peierls $(H, T)$ phase diagram. This would include, e.g., the location of $H_{c}$ and the Lifshitz point $\left(H^{*}, T^{*}\right)$.

(2) An examination of the magnetic thermodynamic properties, in particular the specific heat, for anomalies due to soliton states.

(3) A comparison of experimentally determined excitation energy gaps with theoretically predicted values, soliton and magnon.

An appreciation of the spectral excitations in a magnetic field should underlie our discussions of tests 1,2 , and 3. Let us consider $T=0$. The critical field $H_{c}$ is determined from the dominant excitation gap which decreases in the presence of an applied magnetic field $H$. At $H=H_{c}$, the field-dependent excitation gap vanishes and the system passes from a phase with a gap to a gapless regime, as noted already above. The equation determining $H_{c}$ is thus

$$
\begin{aligned}
& \Delta E(H)=\Delta E(0)-g \mu_{B} H S_{T}^{z}, \quad H \leq H_{c} \\
& \Delta E(H)=0, \quad H=H_{c} .
\end{aligned}
$$

( $g$ is the Landé $g$ factor and $\mu_{B}$ the Bohr magneton. The quantity $S_{T}^{z}$ is the effective spin of the excitation.) In the spin-exciton picture, in zero field, the ground state is a singlet with $S=0$ and the lowest excitations are $S=1$ triplets. In the presence of a field the relevant energy states are characterized by $S_{T}^{z}=0$ and 1 , respectively. All soliton theories consistently predict that the spin of a soliton is given by $S\left(S_{T}^{z}\right)=\frac{1}{2}$. From Eqs. (2) and (3) we obtain the result

$$
H_{c}^{\mathrm{sol}} \sim 0.6 H_{c}^{\mathrm{mag}}
$$

For a given zero-field excitation gap $\Delta E \equiv \Delta(T \rightarrow 0)$, therefore, the soliton picture predicts a critical field about $60 \%$ of the size of that predicted by the spin-exciton picture. The $T=0$ energetics scheme is summarized in Fig. 7. The spin-exciton (magnon) excitations form the lower boundary of a triplet excitation continuum above the gap $\Delta E^{\mathrm{mag}}$. By contrast, the soliton excitation is a localized excitation which has been split out of the triplet continuum; i.e., a localized midgap state. We now discuss tests $1-3$ in light of Fig. 7.

(a) We have already observed above that experimental data on the three compounds, TTF-BDT(Au), TTF$\operatorname{BDT}(\mathrm{Cu})$, and $\mathrm{MEM}-\left(\mathrm{TCNQ}_{2}\right)$ give a very good quantitative fit to the phase diagram predicted by Cross and Fisher, ${ }^{16}$ particularly as regards the variation of the Lifshitz point $\left(H^{*}, T^{*}\right)$. The existing soliton theories are formulated in the vicinity of $T=0$ and thereby do not directly give predictions for $H^{*}$ and $T^{*}$. However, we observe from Figs. 1 and 4 that $H_{c} \cong H^{*}$, since the first order DI phase boundary is almost horizontal. Hence we obtain a prediction for $H^{*}$ for the soliton picture without having to construct a finite temperature development of the theories. We have already noted that the soliton value of $H_{c} \sim H^{*}$ is considerably lower than the value of Cross and Fisher. Since the Cross-Fisher value fits the experi-

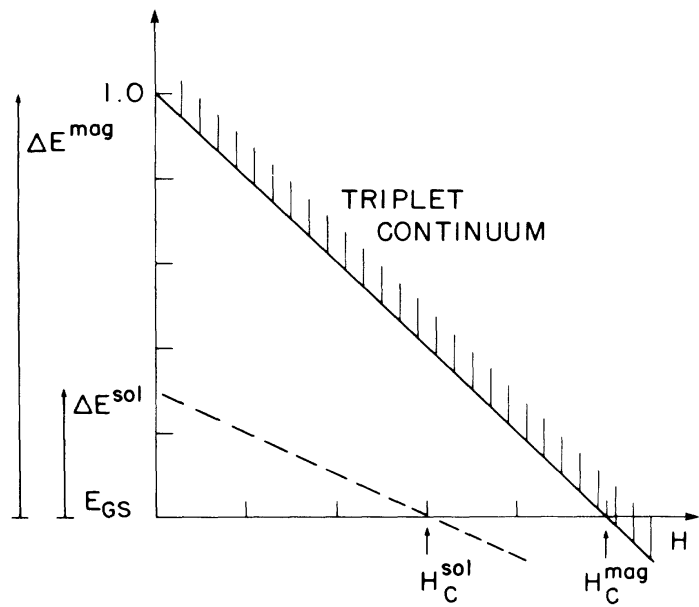

FIG. 7. Schematic view of the excited states of a spin-Peierls system in the presence of a magnetic field. The conventional picture of an alternating chain predicts an excitation gap $\Delta E^{\text {mag }}$, from a singlet ground state $E_{\mathrm{GS}}$ to a triplet excited continuum. The gap $\Delta E^{\mathrm{mag}}$ is field dependent, disappearing at $H_{c}^{\mathrm{mag}}$. More recent soliton theories predict a gap $\Delta E^{\text {sol }}$ to a localized soliton state with spin $-\frac{1}{2}$. $\Delta E^{\text {sol }}$ also decreases linearly with field, disappearing at $H_{c}^{\text {sol }}$.

ment, it follows that the soliton picture does poorly on this particular test.

(b) The localized, low-lying soliton excitation gap given in Eq. (2) and sketched in Fig. 7 should manifest itself in low-temperature Schottky-like structure in the magnetic specific heat, followed at higher temperatures by a broad peak associated with the spin-1 magnon continuum. These features should be distinguished from the cooperative ordering anomaly at $T=T_{\mathrm{SP}}$. No such structure is observed, not only in the case of TTF-BDT(Au) but also for TTF-BDT $(\mathrm{Cu})$, and especially for $\mathrm{MEM}-\left(\mathrm{TCNQ}_{2}\right)$, where data exist down to even lower relative temperatures. ${ }^{32}$

(c) The critical field has been rather accurately determined for all three spin-Peierls compounds. The $T \rightarrow 0$ excitation gaps inferred from Eq. (3), $\Delta^{H}(0)$, may be compared with values $\Delta^{T}(0)$ determined from an analysis of the low-temperature activation energies of the zero-field magnetic specific heat and susceptibilities. Determining the $\Delta^{T}(0)$ was difficult on the basis of existing data, and consistency of values for both $\Delta^{H}(0)$ and $\Delta^{T}(0)$ was not obtained. The zero-field excitation gaps derived for all existing spin-Peierls systems are consistently too large to agree with soliton or spin-exciton theory. The agreement with the soliton picture is, however, worse than with the spin-exciton picture. For the complete resolution of this complex situation, high-quality data on the magnetic thermodynamic properties for all three compounds down to even lower relative temperatures are required.

Hence, to conclude this section, we observe that in the case of all three validity tests, i.e., critical parameters of the global $(H, T)$ phase diagram, low-temperature anomalous structure in the specific heat, and $T \sim 0$ excitation energy gap considerations, the experimental evidence 
favors a spin-exciton picture over a soliton-excitation picture.

The reason for our failure to observe the characteristic features of soliton excitations may reflect the fact, long observed, ${ }^{33}$ that spin-Peierls transitions in real systems show pronounced mean-field character. This could explain the apparent unimportance of nonlinear fluctuations (solitons) and the success of theories which do not include them.

\section{CONCLUSIONS}

A considerable amount of specific heat and susceptibility data, both in zero and nonzero field, is now available for a variety of spin-Peierls compounds. Prominently featured here is the system TTF-BDT(Au), which permits detailed experimental study of all significant regions of the global $(H, T)$ phase diagram. In particular, new high-field specific-heat data demonstrate conclusively the presence of a high-field phase predicted to be incommensurate. The specific-heat data define a high-field (IU) phase boundary in agreement with that derived previously from ac susceptibility measurements, and also are in excellent agreement with the theory of Cross and Fisher. The specific-heat data lay to rest doubts concerning the reliability of $\chi_{\mathrm{ac}}$ data in determining the IU boundary. The reason for doubt is that pronounced relaxation effects in the high-field phase result in striking differences between $\chi_{\mathrm{ac}}$ and $\chi_{\mathrm{dc}}$ in this region.

A theoretical explanation for these differences, and particularly the behavior of $\chi_{\mathrm{dc}}$, is presented in the context of the behavior of an alternating chain as a function of field. ${ }^{25}$ These remarks are fully supported by a mean-field magnetoelastic calculations. ${ }^{30}$

Some discussion is given of the nature of the DI phase boundary in comparison with the familiar first-order phase boundary of a spin-flop system. The unusual hysteretic behavior associated with the DI line is interpreted in terms of a recent theory. ${ }^{23}$

Soliton theories of the spin-Peierls transition have received much attention recently. The soliton predictions for the basic excitations and certain parameters of the $(H, T)$ phase diagram differ greatly from the more traditional spin-exciton, mean-field picture on which our discussions here have essentially been based. These differences allow several experimental tests to determine the relative validity of each type of theory. All tests favor the traditional theories over the newer soliton theories, thus justifying the overall point of view of this paper.

Note: When this paper was in final preparation, we received a copy of work by de Jongh et al., ${ }^{34}$ in which measurements of $\chi_{\mathrm{ac}}$ and $\chi_{\mathrm{dc}}$ were extended to lower temperatures. The new results are consistent with both data and interpretation presented herein. However, the overall theoretical interpretation by de Jongh et al. differs considerably from that presented here.

\section{ACKNOWLEDGMENTS}

We acknowledge stimulating discussions and exchange of information with Jos de Jongh, H. de Groot, F. Greidanus, and other members of the Kamerlingh Onnes Laboratorium. We are also happy to acknowledge useful correspondence with B. Horovitz and valuable discussions with C. Tsallis. We are also deeply grateful to J. W. Bray for useful comments on the various soliton theories and for a careful reading of the manuscript. Financial support has been provided by NSF Grant No. DMR8603036, by NATO, and (for L.V.I.) by AFOSR (AFSC) under Contract No. F49620-79-C-0051.
${ }^{*}$ Present address: Department of Chemistry, Rensselaer Polytechnic Institute, Troy, NY 12181.

${ }^{1} \mathrm{~A}$ comprehensive review of the spin-Peierls transition is given by J. W. Bray, and L. V. Interrante, I. S. Jacobs, and J. C. Bonner, in Extended Linear Chain Compounds, edited by J. S. Miller (Plenum, New York, 1982), p. 353.

2J. W. Bray, L. V. Interrante, I. S. Jacobs, D. Bloch, D. E. Moncton, G. Shirane, and J. C. Bonner, Phys. Rev. B 20, 2067 (1979).

3J. Kondo and T. Kinoshita, J. Phys. Soc. Jpn. 51, 1412 (1982); S. Inagaki and H. Fukuyama, ibid. 52, 3620 (1983).

${ }^{4} Y$. Lépine, Phys. Status Solidi B 102, K15 (1980).

${ }^{5}$ D. Bloch, J. Voiron, J. C. Bonner, J. W. Bray, I. S. Jacobs, and L. V. Interrante, Phys. Rev. Lett. 44, 294 (1980).

${ }^{6}$ I. S. Jacobs, J. W. Bray, H. R. Hart, Jr., L. V. Interrante, J. S. Kasper, D. Bloch, J. Voiron, J. C. Bonner, D. E. Moncton, and G. Shirane, J. Magn. Magn. Mater. 15-18, 332 (1980).

${ }^{7}$ I. S. Jacobs, J. W. Bray, L. V. Interrante, D. Bloch, J. Voiron, and J. C. Bonner, in Physics in One Dimension, edited by $\mathrm{J}$. Bernasconi and T. Schneider (Springer, New York, 1981), p. 173

8J. A. Northby, H. A. Groenendijk, L. J. de Jongh, J. C. Bonner, I. S. Jacobs, and L. V. Interrante, Phys. Rev. B 25, 3215 (1982).

${ }^{9}$ J. A. Northby, F. J. A. M. Greidanus, W. J. Huiskamp, L. J. de
Jongh, I. S. Jacobs, and L. V. Interrante, J. Appl. Phys. 53, 8032 (1982).

${ }^{10}$ T. W. Hijmans, H. B. Brom, and L. J. de Jongh, Phys. Rev. Lett. 54, 1714 (1985).

${ }^{11} \mathrm{D}$. Bloch, J. Voiron, and L. J. de Jongh, in High-Field Magnetism, edited by M. Date (North-Holland, Amsterdam, 1983), p. 19.

${ }^{12}$ L. J. de Jongh, J. Appl. Phys. 53, 8018 (1982).

${ }^{13}$ T. W. Hijmans, W. H. Korving, G. J. Kramer, H. B. Brom, L. J. de Jongh, I. S. Jacobs, and L. V. Interrante, Mol. Cryst. Liq. Cryst. 120, 251 (1985).

${ }^{14}$ E. Pytte, Phys. Rev. B 10, 2039 (1974).

15J. W. Bray, Solid State Commun. 26, 771 (1978); L. N. Bulaevskii, A. I. Buzdin, and D. I. Khomskii, Solid State Commun. 27, 5 (1978).

16M. C. Cross and D. S. Fisher, Phys. Rev. B 19, 402 (1979); M. C. Cross, Phys. Rev. B 20, 4606 (1979).

${ }^{17}$ Y. Takaoka and K. Motizuki, J. Phys. Soc. Jpn. 47, 1752 (1979).

${ }^{18}$ C. Tannous and A. Caillé, Can. J. Phys. 57, 508 (1979). Phys. Status Solidi B 103, K157 (1981); Y. Lépine, Phys. Rev. B 24, 5242 (1981).

${ }^{19}$ M. Grabowsky, K. R. Subbaswamy, and B. Horovitz, Solid State Commun. 34, 911 (1980); B. Horovitz, Phys. Rev. Lett. 46, 742 (1981) 
${ }^{20}$ T. Nakano and H. Fukuyama, J. Phys. Soc. Jpn. 49, 1679 (1980); 50, 2489 (1981).

${ }^{21}$ A. Kotani and I. Harada, J. Phys. Soc. Jpn. 49, 535 (1980); I. Harada and A. Kotani, ibid. 51, 1737 (1982).

${ }^{22}$ A. Aharony and P. Bak, Phys. Rev. B 23, 4770 (1981).

${ }^{23}$ R. A. T. de Lima and C. Tsallis, Phys. Rev. B 27, 6896 (1983).

${ }^{24}$ L. A. Turkevich and S. Doniach, Ann. Phys. (N.Y.) 139, 343 (1982).

${ }^{25}$ M. Mohan and J. C. Bonner, J. Appl. Phys. 53, 8035 (1982).

${ }^{26}$ U. M. Diederix, H. W. J. Blöte, J. P. Green, T. O. Klaassen, and N. J. Poulis, Phys. Rev. B 19, 420 (1979).

${ }^{27}$ J. C. Bonner, S. A. Friedberg, H. Kobayashi, D. L. Meier, and H. W. J. Blöte, Phys. Rev. B 27, 248 (1983).

${ }^{28}$ This general effect is observed also in uniaxially anisotropic
Ising-Heisenberg antiferromagnetic chains; see C. N. Yang and C. P. Yang, Phys. Rev. 151, 258 (1966).

${ }^{29}$ Perhaps a vertical tangent at the IU transition points; see Fig. 5 of Ref. 26.

${ }^{30}$ M. Fujita and K. Machida, J. Phys. Soc. Jpn. 53, 4395 (1984).

${ }^{31}$ The value of the constant varies slightly in different soliton theories from 0.28 to $1 / \pi$.

${ }^{32}$ S. Huizenga, J. Kommandeur, G. A. Sawatzky, B. T. Thole, K. Kopinga, W. J. M. de Jonge, and J. Roos, Phys. Rev. B 19, 4723 (1979).

${ }^{33}$ J. W. Bray and S.-T. Chui, Ferroelectrics 17, 387 (1977).

${ }^{34}$ L. J. de Jongh, H. B. Brom, H. J. M. de Groot, T. W. Hijmans, and W. H. Korving, J. Magn. Magn. Mater. 54-57, 1447 (1986). 